\title{
Free-standing Notch Filter Image Masks for Coronagraphy
}

\author{
J. Crepp ${ }^{1}$, J. Ge ${ }^{1}$, S. Miller ${ }^{1}$, D. McDavitt ${ }^{1}$, A. Vanden Heuvel ${ }^{1}$, \\ and M. Kuchner ${ }^{2}$ \\ ${ }^{1}$ Astronomy Department, University of Florida \\ Bryant Space Science Center, P.O. Box 112055 \\ Gainesville, FL 32611-2055 \\ contact email: jcrepp@astro.ufl.edu \\ ${ }^{2}$ Exoplanets \& Stellar Astrophysics Laboratory \\ NASA/Goddard Space Flight Center \\ Greenbelt, MD 20771, USA
}

\begin{abstract}
The Lyot coronagraph can achieve significant on-axis starlight suppression when equipped with a notch filter image mask. Notch filter masks are placed in the focal plane and have small intricate features. They must be manufactured using nanofabrication techniques, when designed for coronagraphs operating at visible or near infrared wavelengths. This process often involves depositing an opaque material onto a glass substrate whereby a pattern is then etched. The substrate however can be a source of internal reflections and chromatic aberrations that limit the coronagraph's dynamic range and broadband performance. We present theoretical and experimental work on a new series of image masks that do not require the support of a glass substrate. We show that by adding periodic bar structures of appropriate spacing, it may be possible to manufacture a Silicon binary notch filter mask that is "free-standing".
\end{abstract}

Keywords. extrasolar planets — high contrast imaging - coronagraphy — terrestrial planet finder - circumstellar matter.

\section{Introduction}

The detection of terrestrial exoplanets orbiting in the habitable zone of nearby stars will require an instrument with unprecedented wavefront stability and diffraction control. Additionally, the instrument must have a large collecting area, a broad bandwidth, and the ability to adaptively correct for imperfections in optical components that scatter light. At large contrast ratios, these capabilities correspond to tight tolerances. Subtle chromatic effects could easily limit the instrument's dynamic range and broadband performance.

One of the leading designs for the Terrestrial Planet Finder Coronagraph (TPF-C) is a Lyot coronagraph that is equipped with an 8th-order band-limited or notch filter image mask (Kuchner, Crepp, \& Ge 2005). These masks perform well in theory and in practice. They have high off-axis throughput $(\sim 40 \%)$, a large search area, and a broad bandwidth (Kuchner \& Traub 2002, Kuchner \& Spergel 2003). They also provide resistance to loworder aberrations (Shaklan \& Green 2005, Crepp et al. 2005), and have enabled the Lyot coronagraph to achieve the deepest contrast in the lab to date (Trauger et al. 2004).

Binary versions of these masks are manufactured by depositing an opaque material such as Chromium, Aluminum, or Gold onto a glass substrate; then a pattern is "etched out", and an anti-reflective coating is applied (Debes et al. 2004). The glass provides mechanical support, but is a source of unwanted internal reflections and chromatic aberrations. In this study, we aim to significantly reduce these effects in the image mask by eliminating the need for the glass all together. 

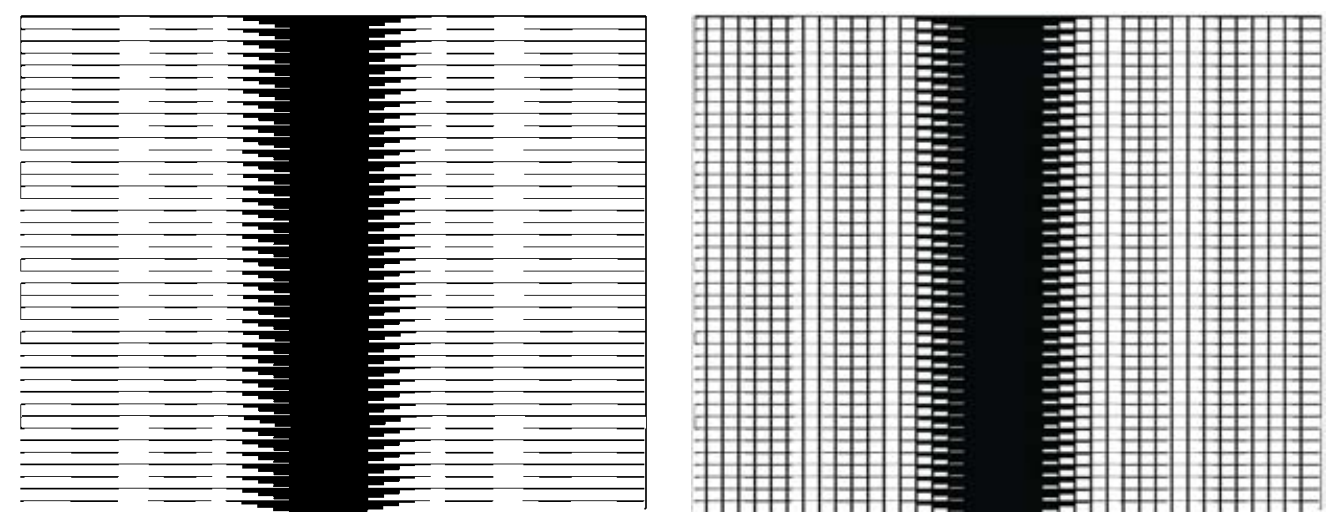

Figure 1. Simulated image of a sampled binary 8th-order notch filter image mask (left), and a free-standing mask equivalent (right). For a small penalty in off-axis attenuation, free-standing masks offer a way to circumvent the need for glass substrates.

Figure 1 displays a simulated image of a sampled binary 8th-order notch filter mask and a "free-standing" mask equivalent. The periodic bar structures in the free-standing mask provide strength in the absence of glass, and prevent the mask from collapsing under it's own weight, or from any external forces such as the harsh jostling of a shuttle lift-off. These structures clearly increase the off-axis attenuation of the coronagraph. However, depending on their breadth, the broadband performance of the masks may justify the loss in throughput. We intend to directly measure these quantities in the lab.

It is possible to model the performance of free-standing masks numerically, however a full electromagnetic treatment is required. The size of the smallest features are smaller than the wavelength of light, therefore it is necessary to include polarization, the finite thickness of mask, and other complications. Although such simulations are useful, the results must be confirmed experimentally. Moreover, it is not clear how narrow one can make the bar structures before they are no longer supportive. The contrast, wavelength dependence, and throughput of the mask are all functions of the mask thickness, width of the bars, and kind of material that is used, as well as the $f / \#$ of the system.

In the next section, we describe the basic theory for how binary free-standing masks diffract light to first order. Then we provide a summary of our nanofabrication techniques and progress towards constructing the first prototype.

\section{Theory}

Free-standing masks are notch filter masks that are multiplied by a dirac-comb filter of finite width. We use the notation of Kuchner \& Spergel 2003 to describe how these masks interact with light in the image plane.

The equation describing a linear binary mask with vertical support bars is:

$$
\begin{aligned}
\hat{M}(x, y)= & \sum_{n} \delta\left(y-n \frac{\lambda_{\text {min }}}{D}\right) * \hat{M}_{\text {stripe }}(x, y) \times \\
& \left\{\sum_{m} \delta(x-m \Delta x) *\left[1-\Pi\left(\frac{x}{a} \frac{D}{\lambda_{\min }}\right)\right]\right\}
\end{aligned}
$$

where

$$
\Pi\left(\frac{x}{a} \frac{D}{\lambda_{\min }}\right)= \begin{cases}1 & \text { for }|x| \leqslant \frac{a}{2} \frac{\lambda_{\min }}{D} \\ 0 & \text { elsewhere }\end{cases}
$$


and

$$
\hat{M}_{\text {stripe }}(x, y)= \begin{cases}1 & \text { where } y<\hat{M}_{n o t c h}(x) \frac{\lambda_{\min }}{D} \\ 0 & \text { elsewhere. }\end{cases}
$$

The factor $\sum \delta(x-m \Delta x) *\left(1-\Pi\left(x D / a \lambda_{m i n}\right)\right)$ is a dirac-comb filter convolved with a tophat. It represents a series of vertical bars, where $\Delta x$ is their separation, and $0 \leqslant a \leqslant 1$ is the width of each individual bar, in units of $\lambda_{\text {min }} / D$. The function $\hat{M}_{n o t c h}(x)$ is a one-dimensional notch filter function that can be either smooth or sampled.

Notch filter masks are designed such that only the low-spatial frequencies, $|u| \leqslant \frac{\epsilon}{2} \frac{D}{\lambda_{\max }}$, diffract light inside the Lyot stop - this light interferes and identically cancels. Higher spatial frequencies $|u| \geqslant\left(1-\frac{\epsilon}{2}\right) \frac{D}{\lambda_{m} a x}$ diffract light outside the Lyot stop - this light is blocked by a stop in the subsequent pupil plane. In theory, the combination can yield infinite dynamic range for an unobstructed entrance aperture.

For free-standing masks, we can choose a spacing for the vertical stripes such that only one term diffracts light inside the Lyot stop and the rest diffract light outside. The Fourier transform of Equation 2.1 is

$$
\begin{aligned}
M(u, v)= & \sum_{n} \delta\left(v-n \frac{D}{\lambda_{\text {min }}}\right) \int \operatorname{sinc}\left(\pi v \frac{\lambda_{\text {min }}}{D} \hat{M}_{\text {notch }}(x)\right) \\
& \times \hat{M}_{n o t c h}(x) e^{-i 2 \pi u x} d x \\
& * \frac{1}{\Delta x} \sum_{m} \delta\left(u-m \frac{1}{\Delta x}\right) \\
& \times \frac{\lambda_{\text {min }}}{D}(\delta(u)-a \operatorname{sinc}(\pi a u)),
\end{aligned}
$$

where $m, n$ run over all integers. If $\Delta x \leqslant \lambda_{\text {min }} / D$, then only the $u=0$ and $v=0$ terms contribute, and at low-spatial-frequencies Equation 2.4 reduces to

$$
\begin{aligned}
M(u, v) & =(1-a) \delta(u) * M_{n o t c h}(u) \\
& =(1-a) M_{n o t c h}(u) .
\end{aligned}
$$

Therefore, free-standing masks diffract light just like notch filter masks, with the exception that off-axis sources are attenuated by an additional factor of $(1-a)^{2}$. The binary support bars are the mathematical equivalent of a neutral density filter.

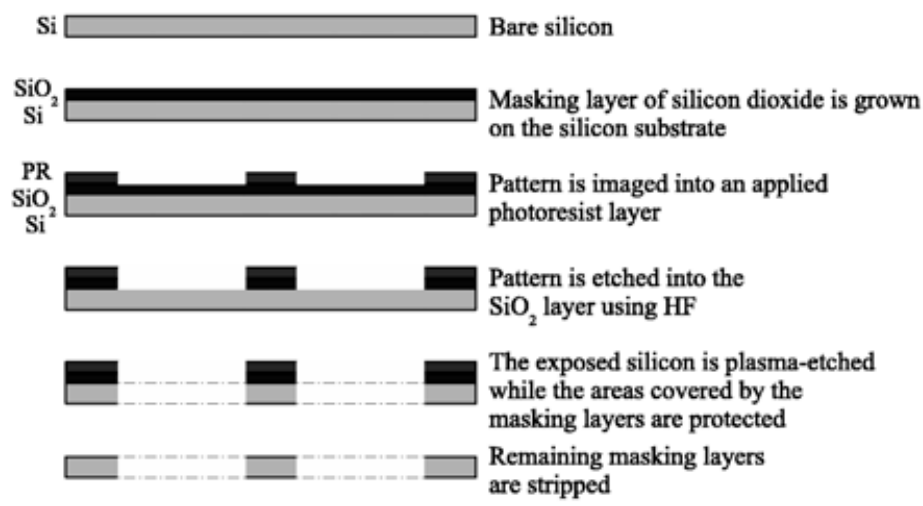

Figure 2. Edge-on view of the nanofabrication process for creating a free-standing mask.

It is also possible to add horizontal stripes. With a clever starting point, these stripes can be made to overlap or "hide" the smallest features in the mask. Of course, the off-axis throughput then goes as $\sim(1-a)^{2}(1-b)^{2}$, where $b$ is the width of each horizontal stripe. 

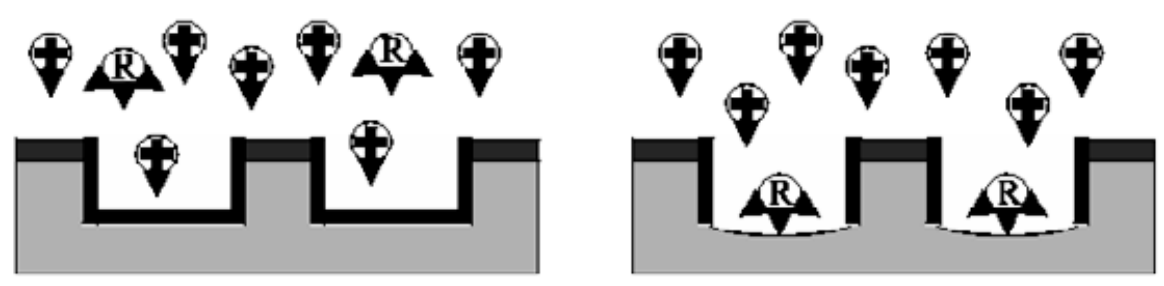

Figure 3. Edge-on view of the ASE process showing the ions selectively stripping the protecting Fluorocarbon layers along the trench bottoms, and the reactive species of the plasma etching away the exposed Silicon.

\section{Nanofabrication: strategy \& progress}

We have chosen Silicon as the base structure for the masks. Silicon is opaque to light with $\lambda \lesssim 900 \mathrm{~nm}$ and can be easily machined into microstructures with nanometer level surface roughness. Additionally, Silicon has a Young's Modulus of $131 \mathrm{GPa}$, which is greater than steel.

Preliminary patterns are being fabricated to determine the minimum allowable dimensions that can be "free standing", the maximum separation of these features, and what level of uniformity and roughness that can be achieved. Once these defining parameters are determined, we will fabricate a fully developed mask, similar to that shown in Figure 1. Our initial experiments are focused primarily on creating square-groove features with vertical sidewalls; although, it may be possible to direct unwanted diffracted light, created by the finite thickness of the masks, away from the optical axis with angled trenches. We will explore this option by experimenting with fast etch rates that compromise anisotropy.
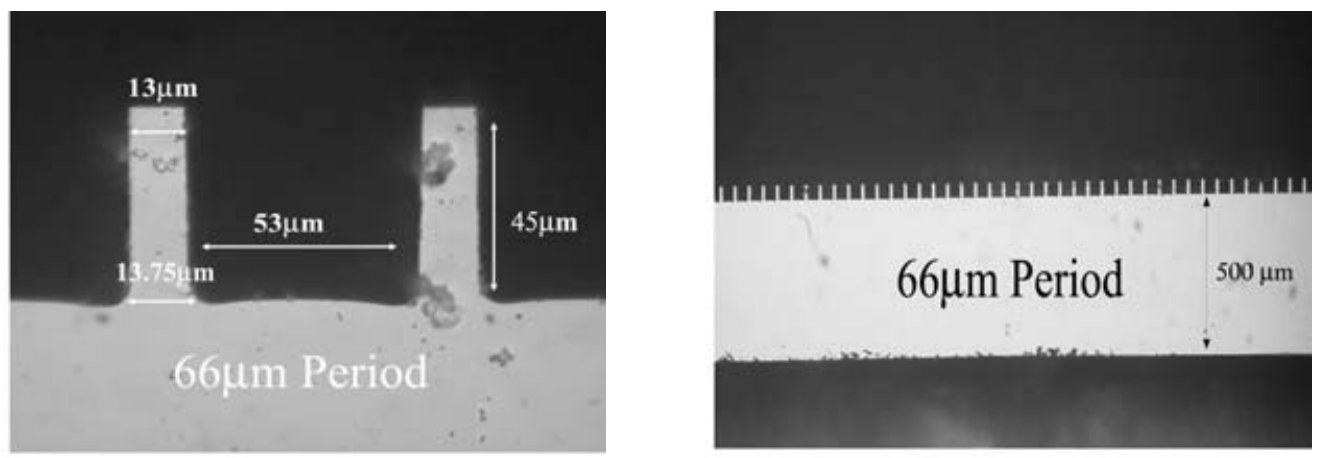

Figure 4. Microscope image of edge-on view of mask that has been partially etched. The goal is to etch all the way through the wafers and to maintain small structural support bars with minimal defects.

The process for fabricating free-standing masks involves the following steps and is illustrated in Figure 2. First, an etch-resistant $\mathrm{SiO}_{2}$ layer is grown on the silicon substrate. Photolithography is then used to transfer the desired pattern from an electron-beam developed mask to the wafer surface with selected areas exposing the oxide layer. Currently we use contact photolithography to produce micron features. Electron-beam direct writing has a resolution on the order of $20 \mathrm{~nm}$, and will also be used. The bare oxide is then wet etched with Hydrofluoric acid down to the underlying Silicon. Using the Advanced Silicon Etch (ASE) process with a Surface Technology Systems - Deep Reactive 
Ion Etcher, plasma etches the pattern down through the Silicon wafer creating our free standing structures. The ASE process is detailed below. Finally, the remaining masking layer is removed and the substrate is cleaned.

The ASE process, based on the original "Bosch Process", anisotropically etches Silicon through a series of alternating passivation and etch steps, producing vertical sidewalls. The process is shown in Figure 3. The etch is free of the constraining geometries of silicon's crystallography that standard wet chemical etching is bound by. Each process cycle starts with a passivation step, where a $\mathrm{C}_{4} \mathrm{~F}_{8}$-based plasma deposits a few Fluorocarbon polymer monolayers across surfaces exposed to the plasma. An isotropic etching plasma of $\mathrm{SF}_{6}$ is then introduced. Ions bombard the surface of the wafer, selectively removing the polymer layers. The plasma, having an increased ion energy in the vertical direction, removes the Fluorocarbon from surfaces parallel to the wafer surface, leaving an exposed silicon surface at the base of trenches. The reactive Fluorine-based species of the plasma isotropically etches the bare Silicon while the remaining Fluorocarbon polymer protects the vertical sidewalls from lateral etching. The cycle is repeated to produce vertical sidewall profiles of $90^{\circ} \pm 2^{\circ}$ through a silicon wafer. Feature sizes can range from 1 to $>500 \mu \mathrm{m}$ and etch depths range from $10-800 \mu \mathrm{m}$ while maintaining aspect ratios of up to 30 .

Several error sources from the process have to be considered. These include: scallops, etch defects that form along the sidewalls created by the cycling of passivation and etching, "grass" that is observed as pillars of silicon at the bottom of trenches, microloading, the etch rate dependence on the aspect ratio, and finally RIE lag, where larger features locally decrease the number of etching species at the expense of narrower features and non-uniformity in the etch rate with respect to the location on the substrate.

\section{Conclusions}

We have discussed the potential utility of a free-standing notch filter image mask for coronagraphy, particularly for use with aggressive space missions such as TPF. We then derived the first order theory for how such masks diffract light: free-standing masks behave like notch filter masks with the exception that they attenuate off-axis sources by a small factor that is a function of the support bar widths. We then outlined our strategy and progress in making the first prototype mask using nanofabrication techniques.

"We acknowledge the support from NSF with grants NSF AST-0451407 and AST0451408, NASA with grants NNG05G321G and NNG05GR41G, JPL TPF program, UCF-UF SRI program, UF and Penn State."

\section{References}

Crepp, J. R., Vanden Heuvel, A. D., Ge, J., Miller, S. P., \& Kuchner, M. J. Laboratory Testing of a Lyot Coronagraph Equipped with an 8th-order Notch Filter Image Mask, submitted to ApJ

Debes, J. H., Ge, J., Kuchner, M. J., \& Rogosky, M., ApJ, 608, 1095-1099, 2004

Kuchner, M. J., Crepp, J. C., \& Ge, J., ApJ, 628, 466-473, 2005

Kuchner, M. J. \& Spergel, D. N., ApJ, 594, 617-626, 2003

Kuchner, M. J. \& Traub, W. A., ApJ, 570, 900-908, 2002

Shaklan, S. B. \& Green, J. J., ApJ, 628, 474-477, 2005

Trauger, J. et al., Proc. SPIE, 5487, 1330-1336, 2004 

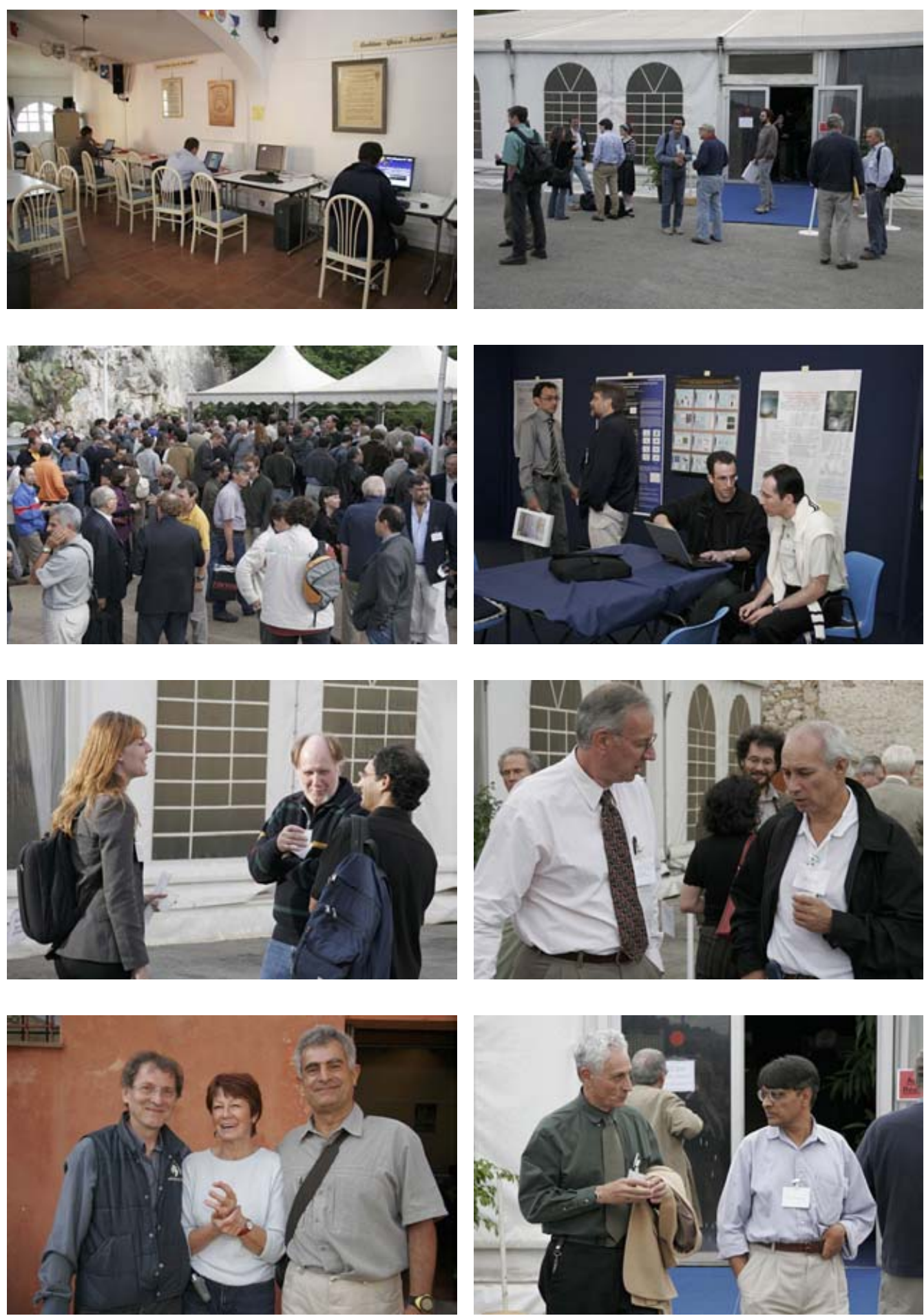

All photographs: Laurent Thareau [1.thareau@free.fr]. 